\title{
The causality between the serum uric acid level and stroke
}

\author{
Ryusuke $\mathrm{Ae}^{1} \cdot$ Mehmet Kanbay $^{2} \cdot$ Masanari Kuwabara $^{3}$
}

Received: 19 September 2019 / Revised: 24 September 2019 / Accepted: 24 September 2019 / Published online: 27 January 2020

(c) The Japanese Society of Hypertension 2020

Serum uric acid (SUA) is well known to be associated with cardiovascular risk factors such as hypertension, obesity, metabolic syndrome, dyslipidemia, diabetes mellitus, insulin resistance, chronic kidney disease, etc [1]. However, it is difficult to show the causality between the SUA level and cardiac and cerebrovascular diseases because the former is easily affected by many confounding factors, such as food, alcohol, medication, and so on [1]. For example, a high calorie intake causes obesity and dyslipidemia, as well as hyperuricemia. Obesity and dyslipidemia themselves are known risk factors for cardiac and cerebrovascular diseases, and therefore, we cannot assess whether hyperuricemia itself is a risk factor for cardiac and cerebrovascular diseases. Many epidemiological studies conducted multiple adjustments to reduce the confounding factors, but epidemiological studies do not prove their causality because these adjustments were not able to exclude confounding completely [2]. The causality between SUA and gout is well established, but the causality between SUA and stroke is still to be discussed, as well as that for hypertension [3] or chronic kidney disease [4].

Several meta-analysis studies have indicated that the SUA level is "independently" associated with stroke $[5,6]$. Kim S.Y. et al. reported that hyperuricemia was associated with a significantly higher risk of both stroke incidence (relative risk (RR) 1.41) and mortality (RR 1.36) in their metaanalyses of unadjusted study estimates. Moreover, after adjusting for known risk factors such as age, hypertension, diabetes mellitus, and cholesterol, hyperuricemia was

Masanari Kuwabara

kuwamasa728@gmail.com

1 Division of Public Health, Center for Community Medicine, Jichi Medical University, Tochigi, Japan

2 Division of Nephrology, Department of Internal Medicine, Koç University School of Medicine, Istanbul, Turkey

3 Intensive Care Unit and Department of Cardiology, Toranomon Hospital, Tokyo, Japan significantly associated with both stroke incidence (RR 1.47) and mortality (RR 1.26) [5]. In this meta-analysis, the results did not differ significantly by sex. Another meta-analysis study by Zhong et al. showed similar results: elevated SUA levels were significantly associated with a modestly increased risk of stroke in both men (RR 1.10 per $1 \mathrm{mg} / \mathrm{dL}$ increase in SUA) and women (RR 1.11) (Fig. 1) [6]. There seems to be no doubt about the relationship between SUA and stroke. However, some epidemiological studies globally reported inconsistent findings on the relationship between the SUA level and stroke [7-9]; therefore, SUA may not be considered an obvious risk factor for stroke. Even among studies focusing only on a Japanese population, some studies suggested that SUA is an independent predictor of stroke $[7,8]$, but others reported negative results $[9,10]$. These inconsistent findings may be derived from different study settings and participants. Moreover, some studies showed that the relationship between the SUA level and stroke risk differed by sex, indicating a nonlinear (J-shaped) relationship in men, whereas an almost linear trend was observed in women (Fig. 1) [6, 10]. The relationship between the SUA level and stroke is well established, but the causality remains controversial because robust evidence has not been adequately accumulated.

The intriguing and timely article by Jiaqi et al. in the present issue of Hypertension Research provided additional evidence of the relationship between the SUA level and stroke [11]. Jiaqi et al. conducted a prospective cohort study using the data from the Circulatory Risk in Communities Study in a general Japanese population, and the results showed that an elevated SUA level is an independent predictor for total and ischemic strokes in women but not in men in a general Japanese population [11]. The strong point of the study is that they conducted multiple adjustments for potential cardiovascular-related factors, not just for age, community, body mass index, smoking and alcohol intake status, systolic blood pressure, atrial fibrillation, serum total cholesterol, triglycerides, estimated glomerular filtration rate, diabetes mellitus, and antihypertensive medication use but also menopausal status in women. Female hormones 
Serum uric acid and risk of stroke in men

A Uric acid and risk of stroke, per $1 \mathrm{mg} / \mathrm{dl}$ increase

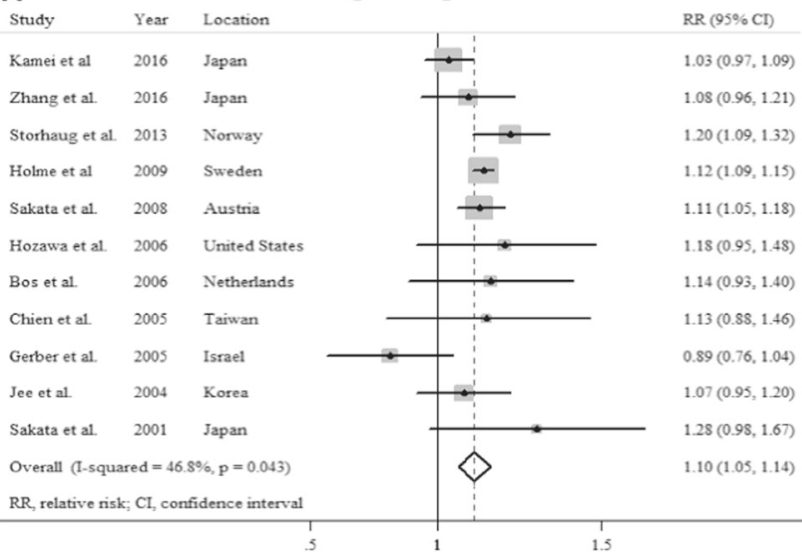

B Uric acid and risk of stroke, nonlinear dose-response

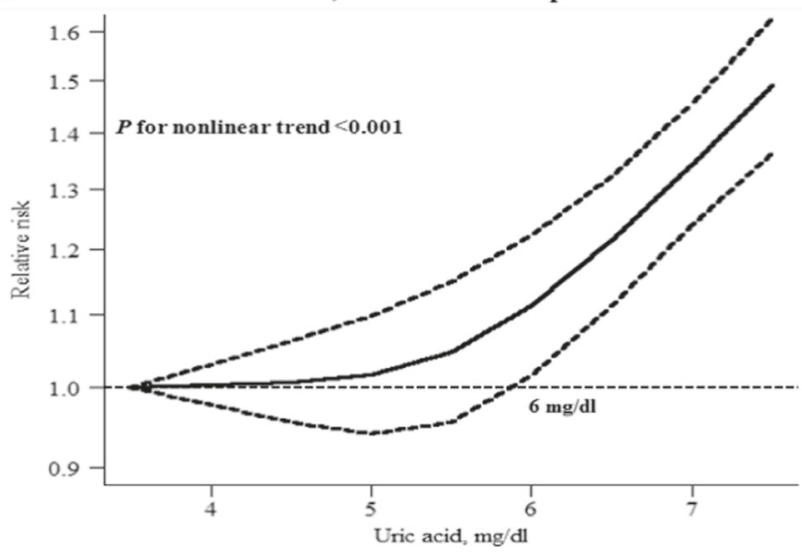

Serum uric acid and risk of stroke in women

A Uric acid and risk of stroke, per $1 \mathrm{mg} / \mathrm{dl}$ increase

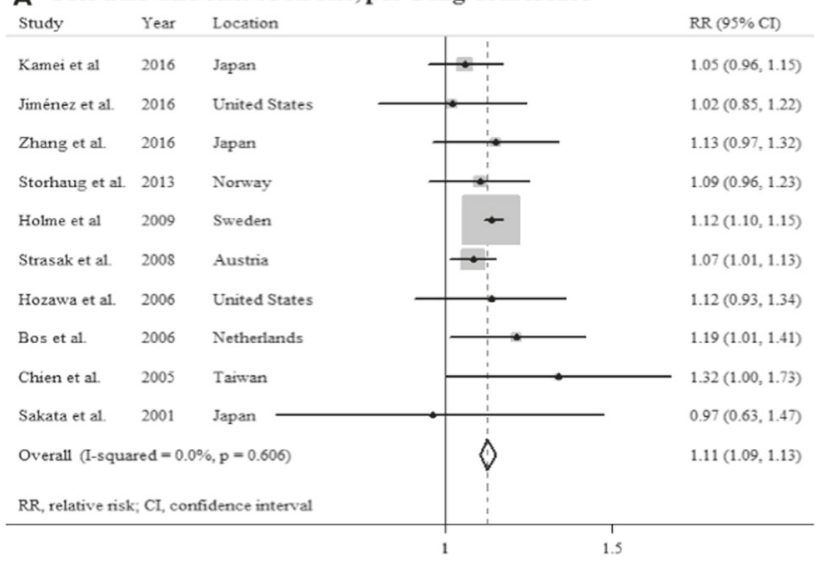

$B$ Uric acid and risk of stroke, nonlinear dose-response

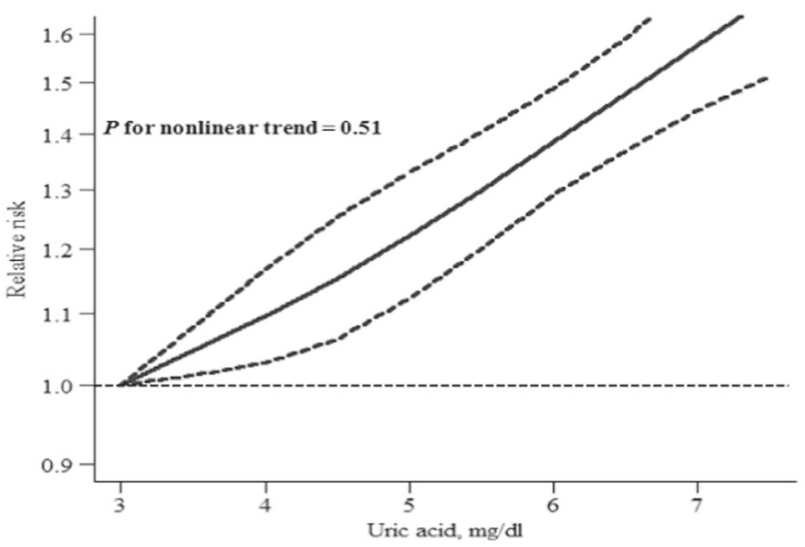

Fig. 1 Serum uric acid and risk of stroke among men and women [6]. a RR relative risks per $1 \mathrm{mg} / \mathrm{dL}$ increase in serum uric acid level. b Nonlinear dose response

decrease SUA levels, and the SUA level in women increases with aging after menopause. The authors checked and adjusted menopausal status in the study to reduce the hormonal effects, which is one of the strongest points of this study. Although Jiaqi et al. failed to show that an elevated SUA level is an independent predictor for total and ischemic strokes in men, they showed a J-shaped risk trend in men, which is consistent with the results from a recent meta-analysis (Fig. 1) [6]. Many reports about the sex and age differences in SUA have been published $[12,13]$. We must account for sex and age differences when conducting studies associated with SUA. The findings by Jiaqi et al. are informative; however, as the authors stated, the study contains certain issues that should be addressed in further studies. The primary issue is the accuracy of the exposure information, although this issue may apply to any other cohort studies. In their study, SUA was measured at one point (at baseline) only and followed up over 20 years. Longer follow-up periods may contribute to a larger effect of other potential factors strongly associated with the development of stroke. The SUA level may vary over time. Further studies are warranted that should be designed to accurately obtain information on the SUA exposure level, which should be regularly monitored. Another issue is that the authors should discuss why their findings were inconsistent with the previous findings that were obtained using a general Japanese population [9]. Both cohorts were recruited from the general Japanese population and included almost the same numbers.

How does SUA independently contribute to the development of stroke? The mechanism remains elusive. Previous studies have suggested that potential mechanisms include enhancing lipid peroxidation and platelet adhesiveness, stimulating vascular smooth cell proliferation, causing vascular inflammation, damaging endothelial cells, and accelerating atherosclerosis [14-17]. Based on this evidence, further studies should be conducted to clarify the relationship between the SUA level and stroke risk. The observational study design (cohort study) is difficult to show the causality between SUA and stroke, even with the 
implementation of multiple adjustments. Further intervention studies on whether SUA lowering treatment can reduce the event of stroke are warranted.

\section{Compliance with ethical standards}

Conflict of interest Dr Kuwabara reports a research grant from Toranomon Hospital, the Okinaka Memorial Institute for Medical Research, and the Gout Research Foundation in Japan. The remaining authors have nothing to disclose.

Publisher's note Springer Nature remains neutral with regard to jurisdictional claims in published maps and institutional affiliations.

\section{References}

1. Kuwabara M. Hyperuricemia, cardiovascular disease, and hypertension. Pulse. 2016;3:242-52.

2. Johnson RJ. Finding the truth: multivariable analysis and the assassination of Abraham Lincoln. J R Coll Physicians Edinb. 2018;48:153-4.

3. Kuwabara M, Hisatome I, Niwa K, Hara S, Roncal-Jimenez CA, Bjornstad $\mathrm{P}$, et al. Uric acid is a strong risk marker for developing hypertension from prehypertension: a 5-year Japanese Cohort Study. Hypertension. 2018;71:78-86.

4. Sato Y, Feig DI, Stack AG, Kang DH, Lanaspa MA, Ejaz AA, et al. The case for uric acid-lowering treatment in patients with hyperuricaemia and CKD. Nat Rev Nephrol. 2019. https://doi.org/ 10.1038/s41581-019-0174-z.

5. Kim SY, Guevara JP, Kim KM, Choi HK, Heitjan DF, Albert DA. Hyperuricemia and risk of stroke: a systematic review and metaanalysis. Arthritis Rheum. 2009;61:885-92.

6. Zhong C, Zhong X, Xu T, Xu T, Zhang Y. Sex-specific relationship between serum uric acid and risk of stroke: a doseresponse meta-analysis of prospective studies. J Am Heart Assoc. 2017;6:pii: e005042.
7. Hozawa A, Folsom AR, Ibrahim H, Nieto FJ, Rosamond WD, Shahar E. Serum uric acid and risk of ischemic stroke: the ARIC Study. Atherosclerosis. 2006;187:401-7.

8. Kamei K, Konta T, Hirayama A, Ichikawa K, Kubota I, Fujimoto $\mathrm{S}$, et al. Associations between serum uric acid levels and the incidence of nonfatal stroke: a nationwide community-based cohort study. Clin Exp Nephrol. 2017;21:497-503.

9. Sakata K, Hashimoto T, Ueshima H, Okayama A, Group NDR. Absence of an association between serum uric acid and mortality from cardiovascular disease: NIPPON DATA 80, 1980-1994. National Integrated Projects for prospective observation of noncommunicable diseases and its trend in the aged. Eur J Epidemiol. 2001;17:461-8.

10. Zhang W, Iso H, Murakami Y, Miura K, Nagai M, Sugiyama D, et al. Serum uric acid and mortality form cardiovascular disease: EPOCH-JAPAN Study. J Atheroscler Thromb. 2016;23: 692-703.

11. Li J, Muraki I, Imano H, Cui R, Yamagishi K, Umesawa M, et al. Serum uric acid and risk of stroke and its types: the Circulatory Risk in Communities Study (CIRCS). Hypertens Res. 2020. https://doi.org/10.1038/s41440-019-0385-5.

12. Lee SW, Kim HC, Nam C, Lee HY, Ahn SV, Oh YA, et al. Agedifferential association between serum uric acid and incident hypertension. Hypertens Res. 2019;42:428-37.

13. Kuwabara M, Hisatome I. Gender difference in the association between uric acid and atrial fibrillation. Circ J. 2018;83:27-29.

14. Kanellis J, Kang DH. Uric acid as a mediator of endothelial dysfunction, inflammation, and vascular disease. Semin Nephrol. 2005;25:39-42.

15. Li P, Zhang L, Zhang M, Zhou C, Lin N. Uric acid enhances PKC-dependent eNOS phosphorylation and mediates cellular ER stress: A mechanism for uric acid-induced endothelial dysfunction. Int J Mol Med. 2016;37:989-97.

16. Montalcini T, Gorgone G, Gazzaruso C, Sesti G, Perticone F, Pujia A. Relation between serum uric acid and carotid intimamedia thickness in healthy postmenopausal women. Intern Emerg Med. 2007;2:19-23.

17. Ruggiero C, Cherubini A, Ble A, Bos AJ, Maggio M, Dixit VD, et al. Uric acid and inflammatory markers. Eur Heart J. 2006;27:1174-81. 